Recepción: 04/07/2018

Aceptación: 25/09/2018

Publicación: 05/10/2018

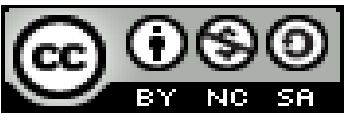

Ciencias de la educación

Artículo de investigación

\title{
El uso de la tecnología como una herramienta pedagógica en los procesos de integración curricular
}

The use of technology as a pedagogic tool in curricular integration processes

\section{Uso da tecnologia como ferramenta pedagógica nos processos de integração curricular}

\author{
Leopoldo V. Venegas-Loor ${ }^{\mathrm{II}}$ \\ ingvenegasloor@gmail.com
}

Paola Y. Moreira-Aguayo ${ }^{\text {II }}$

paola.moreira@unesum.edu.ec

Correspondencia: ingvenegasloor@gmail.com

\footnotetext{
${ }^{\text {I } M a g i ́ s t e r ~ e n ~ E v a l u a c i o ́ n ~ y ~ A u d i t o r i a ~ d e ~ S i s t e m a s ~ T e c n o l o ́ g i c o s, ~ I n g e n i e r o ~ e n ~ C o m p u t a c i o ́ n ~ y ~ R e d e s, ~ D o c t o r a n d o ~ e n ~ E d u c a c i o ́ n ~ e n ~}$ la Universidad Católica Andrés Bello, Caracas, Venezuela; Docente de la Universidad Estatal del Sur de Manabí, Jipijapa, Ecuador.

II Magíster en Enseñanza del Idioma Inglés, Licenciada en Ciencias de la Educación Especialidad Inglés, Doctorando en Educación en la Universidad Católica Andrés Bello, Caracas, Venezuela; Docente de la Universidad Estatal del Sur de Manabí, Jipijapa, Ecuador.
} 


\title{
Resumen
}

Existen factores que avalan positivamente el uso de las tecnologías como una herramienta pedagógica de la integración curricular, entre estos el de potenciar el aprendizaje, pero a la vez existe una gran diversidad de variables que estarían afectando para alcanzar una real efectividad e integración de éstas al currículo, algunas de ellas relacionadas con la capacitación de profesores, equipamiento, gestión de las instituciones educativas e innovaciones, entre otros. Con base en lo expuesto, este ensayo describe y analiza el estado actual de Integración curricular y las Tecnologías de la información y la comunicación (TIC) en el sistema educativo, determinando si existe correlación entre el dominio de competencias TIC y la integración curricular de TIC, para intencionar oportunidades de aprendizajes de calidad en los estudiantes. Del mismo, se pudo concluir que los avances en la tecnología y el uso del Internet dan herramientas educativas que ayudan a enseñar a los estudiantes y satisfacer las necesidades que nunca antes de la era de la revolución informática se lograban satisfacer.

Palabras clave: TIC; herramienta pedagógica; integración curricular y educación.

\begin{abstract}
There are factors that positively support the use of technology as a pedagogical tool for curriculum integration, among these the enhance learning, but at the same time there is a wide range of variables that would be affecting to reach a actual effectiveness and integration of these into the curriculum, some of them related to the training of teachers, equipment, management of educational institutions and innovations, among others. Based on the above, this essay describes and analyzes the current state of curricular integration and the technologies of information and communication (technology ICT) in education, determining if there is a correlation between the domain of ICT skills and integration curriculum ICT, for intentional quality in student learning opportunities. One could conclude that advances in technology and the use of the Internet are educational tools that help teach students and the needs that never before the era of the information revolution are managed to meet.
\end{abstract}

Key words: ICT; pedagogical tool; curriculum integration and education. 


\section{Resumo}

$\mathrm{O}$ uso da tecnologia como uma ferramenta pedagogicExisten fatores que sustentam positivamente o uso de tecnologias como uma ferramenta educacional para a integração curricular, entre estes melhorar a aprendizagem, ainda existe uma ampla gama de variáveis que poderiam afetar a alcançar real efetividade e integração destes ao currículo, alguns deles relacionados à formação de professores, equipamentos, gestão de instituições educacionais e inovações, entre outros. Com base no exposto, este ensaio descreve e analisa o estado atual da integração curricular e das tecnologias da informação e comunicação (TIC) no sistema educativo, determinando se existe uma correlação entre o domínio das competências TIC e a integração curricular das TIC., a intenção de oportunidades de aprendizagem de qualidade para os alunos. A partir daí, concluiu-se que os avanços na tecnologia e o uso da Internet fornecem ferramentas educacionais que ajudam a ensinar os alunos e a satisfazer as necessidades que nunca antes da era da revolução do computador foram atendidas.

Palavras-chave: TIC; ferramenta pedagógica; integração curricular e educação.

\section{Introducción}

La creciente popularidad del Internet, la ha puesto a la vanguardia de la instrucción y debate sobre tecnología. La disponibilidad de la web y la facilidad de uso han impulsado un enorme aumento en la actividad de aprendizaje a distancia en los últimos años. Desde su concepción, a menudo eclipsado por el hecho de que el aprendizaje a distancia y los programas académicos han utilizado durante mucho tiempo Internet, de una forma u otra. Se proporcionan servidores de protocolo (FTP) y "Gopher" acceso remoto a centrales académicas desarrollando materiales de entrenamiento. E-mail, servidores de listas / grupos de noticias, y capacidades de "retransmisión" o "chat" permitió a los estudiantes comunicarse entre ellos y con sus instructores en un lugar distante. Hasta hace poco, sin embargo, interfaces engorrosas y limitadas de conectividad obstaculizan el uso pedagógico efectivo de estos recursos. Al respecto, cabe señalar que las computadoras no hacen docentes, son excelentes para proporcionar acceso a la información, a ofrecer un servicio flexible de comunicación, incluso simulando experiencias que serían poco práctico para ofrecer en un entorno en vivo. Sin embargo, el corazón de la enseñanza es el diagnóstico de necesidades de aprendizaje, evaluación de los medios y métodos disponibles para 
abordar ellos, y el análisis de las interacciones entre estos factores para prescribir y conducir la instrucción más efectiva mediante intervenciones para cada estudiante. En estas actividades, la computadora lo hará, al menos, en el futuro previsible, sigue siendo un sustituto inferior para un humano calificado como docente.

En los últimos años, sin embargo, muchos de estos obstáculos han desaparecido. El interfaz más intuitivo de apuntar y hacer clic de la Web ha reemplazado el lenguaje arcano de FTP y el directorio de DOS de Gopher. Esto ha masificado el uso de Internet, lo que resulta en una explosión de nuevos sitios y mayor conectividad entre ellos. Estos avances ahora están desbloqueando el potencial educativo de Internet, no para reemplazar los métodos convencionales, pero si para lograr lo que antes era imposible, o para servir a las audiencias que nunca antes fueron accesibles. Este es el precepto central detrás de un currículo efectivo integrado con los recursos que brinda Internet o cualquier otra tecnología de aprendizaje. La disponibilidad de una nueva herramienta educativa como el Internet no representa la solución a todas las herramientas obsoletas, aunque la Web puede ser efectiva para simular ciertas actividades prácticas que de otra manera serian demasiado costosas, peligrosas o desagradables Kinzie, M. (2006). Sobre todo, el uso efectivo de la Web no disminuye el papel central del docente en guiar y facilitar el proceso de aprendizaje.

\section{Desarrollo}

El docente de hoy a menudo habita en un mundo en el que el ruido institucional y el facilismo que lo rodea, le impide hacer un uso correcto de los beneficios que puede llegar a obtener mediante la aplicación eficaz de los medios tecnológicos, usando el Internet con poca orientación sobre cuándo es efectivo y requerido su uso. Los innovadores fueron a menudo entusiasmados con el potencial del Internet que desarrollaron sitios con poca o ninguna experiencia de aprendizaje. Quizás peor, el Internet abrió una frontera ajena a muchos docentes: una "cibercultura" completa con sus propias normas, reglas de interacción, y principios de navegación para los cuales los programas de formación docente no se prepararon para ellos. A menudo, el entrenamiento para su uso efectivo no se mantuvo, dejando docentes hastiados por las primeras experiencias siendo deslumbrantes, pero con un uso ineficaz, o ansioso pero confundido acerca de cómo integrar esta herramienta en la práctica en el aula 
En este ensayo, se ofrece un marco para la integración curricular efectiva del Internet y la Web en particular. Incluyo cinco categorías principales de uso y se presentan algunos ejemplos de su uso efectivo, también se puede encontrar en la educación pública (Friel y Knuth, (2016), Seguin, (2017) y capacitación empresarial (Barron \& Orwig, (2018)). Estas categorías son:

Traer la escuela al mundo,

Trayendo el mundo a la escuela,

Preparación de aprendizaje y seguimiento,

Aprendizaje colaborativo en varios sitios,

Exportación de materiales a otras instituciones

\section{Llevando a la escuela al Mundo}

Esta es probablemente la imagen más común de aprendizaje a distancia. A medida que más estudiantes regresan a las escuelas, buscando avanzar su educación durante sus carreras, su importancia crece Incluso en las escuelas públicas, es común para adultos que no completaron la escuela secundaria para ingresar un diploma o equivalencia La web ofrece una importante herramienta hacia estos fines. Una pequeña parte de esto es tener una computadora conectada a Internet y saber usar la misma, donde un prediseñado software de instrucciones y presente el material, intentando diagnosticar el aprendizaje necesidades, e intenta igualar las intervenciones (de un conjunto predefinido) a estos necesariamente, muchos otros usos son posibles.

Las iniciativas de este tipo a menudo se centran en hacer uso de los recursos y la experiencia de la escuela disponible para estudiantes en ubicaciones remotas. Por ejemplo, los profesores pueden colocar enlaces a folletos de los estudiantes, lectura anticipada, paquetes, presentaciones de PowerPoint y otros materiales de clase en sus cursos en páginas web. Algunas instituciones pilotearon este enfoque incluso antes de la masificación del Internet, lo que permite cambiar el énfasis de la instrucción solamente en el aula y pasar de la teoría, a la aplicación de conceptos en la práctica.

Carver y Biehler, (2014), indican que las primeras aplicaciones pueden ser predominantemente digitalizaciones de materiales de papel convencionales. Los usos más efectivos emplean las capacidades multimedia e hipermedia de la web para facilitar el descubrimiento guiado y para reforzar el material que permita apelar a diferentes estilos de aprendizaje. 
Otro enfoque para este objetivo ha llevado a otras instituciones a usar la Web para hacer trabajos de referencia disponibles al minuto a usuarios remotos. Los mejores ejemplos de esto son sitios dinámicos que ofrecen una mirada en la investigación actual o problemas profesionales en la escuela. Estos sitios permiten que el estudiante distante toque organizaciones y recursos escolares bajo demanda, y para ofrecer comentarios inmediatos relacionando lo que ven con sus experiencias. También pueden ser igualmente útil para mantener a los estudiantes locales (o padres) informados de los acontecimientos de la escuela, y para solicitar comentarios de estos y de otras instituciones dentro de la comunidad.

Una aplicación obvia es un estudio auto-dirigido, mientras esto es útil para algunos temas (como veremos en la sección de preparación de aprendizaje y seguimiento), otros requerirán dirección y orientación. Para esos temas que requieren más interacción o tutoría que el software puede proporcionar, otra solución debe ser encontrada, una opción es un programa de facultad adjunto, que difumina la distinción entre residente y la instrucción no residente. Usando esta estrategia, los estudiantes usan Internet para obtener materiales del curso y apoyo de documentos de la escuela, como se describe más arriba, pero aún proporciona la mayoría de las instrucciones en persona. Estos instructores pueden van desde profesionales completamente certificados hasta tutores bien informados. Bajo tal, un programa, instructores y estudiantes pueden utilizar también otros medios tecnológicos, para conferir con directores de curso o instructores residentes en la escuela cuando necesitan orientación o tutoría adicional. Claramente, esta estrategia es más útil cuando los sitios remotos frecuentemente sirven a muchos estudiantes.

\section{Llevando el mundo al Colegio}

Es natural para los profesores universitarios, buscar métodos para poder llevar su experiencia y enseñar a pesar de la distancia; siendo el Internet una de las herramientas más efectivas para ayudarnos a traer la experiencia del mundo a sus estudiantes. Los estudiantes se benefician enormemente, por ejemplo, de tener una clase teórica sobre la transición del apartheid en Sudáfrica a una conversación con un educador que viva en Sudáfrica y lo esté viviendo. Del mismo modo, una escuela podría invitar a sus alumnos de vuelta, electrónicamente, para hablar sobre los desafíos encontrados dentro del área de trabajo después de la graduación y sus recomendaciones u observaciones sobre los contenidos recibidos en clase. 
Iniciativas básicas de este tipo podrían implicar una consulta con la materia mediante expertos por correo electrónico o enlaces a páginas, lo que les permite hacer presentaciones de expertos en las materias de invitados a clases, con necesidades o intereses comunes para discutir los problemas que enfrentan.

\section{Preparación de aprendizaje y seguimiento}

Esta es una de las posibilidades más emocionantes para el uso del Internet. La mayoría de los cursos que impartimos siempre requerirá algunas interacciones personales. Para algunos, la interacción cara a cara de los participantes es la forma más efectiva para alentar el aprendizaje deseado. Por otros, el crecimiento social y la colaboración que solo son posibles de forma presencial. Sin embargo, la mayoría de los cursos también contienen material que no requiere instrucción cara a cara, o incluso porciones donde la instrucción cara a cara es demostrable

Se podrían utilizar tecnologías de aprendizaje a distancia para proporcionar al estudiante, antes de que comience un curso y después de que termine, dándole URL de las páginas de introducción para los cursos. Desde allí, podrían ver, analizar y estudiar lo que el docente espera de ellos al momento del comienzo del curso. Una prueba previa entregada en la web podría servir como autoevaluación. Aun así, los beneficios de tal preparación guiada al final de la escuela son sustancial. Puede ayudar a estandarizar la habilidad y conocimiento que el estudiante ya posee. Esta es una manera ideal de mostrar cómo el sujeto se une al panorama general del plan de estudios.

\section{Colaboración multi-sitio Aprendizaje}

Cuando las personas piensan en las escuelas, generalmente piensa en lugares físicos. Históricamente, esta era la única forma en que el conocimiento y experiencia de todos los campos podría ser recolectado, estandarizado y llevado a cabo sobre la población estudiantil. A pesar de los beneficios que podemos obtener de las categorías descritas aquí, este modelo generalmente sigue siendo la forma más efectiva de transmitir habilidades complejas y conocimiento que comprende una educación.

No obstante, este enfoque tiene algunas desventajas, los estudiantes enseñados dentro de los límites de sus propias aulas están en una situación donde solo se les nutre de teoría bajo el criterio 
único del docente de la materia, ignorando en muchos casos perspectivas diferentes a las expuestas en clase sin llegar a comprender o analizar otros puntos de vista sobre el tema. Exportación de materiales a otras instituciones

Otro enfoque implica tomar las fortalezas únicas o experiencia de otras escuelas, y aplicarlas dentro de sus propias especialidades. Internet, es una herramienta que está disponible para el mercado de la educación que permite a unidades educativas compartir materiales y experiencias con otras. Las ofertas pueden variar desde seminarios de sus expertos a cursos completos combinando estos y otros recursos.

\section{Conclusiones}

Los avances en la tecnología y el uso del Internet dan herramientas educativas que ayudan a enseñar a los estudiantes y satisfacer las necesidades que nunca antes de la era de la revolución informática se lograban satisfacer. Gran parte de los cursos ahora se imparten en forma presencial en las escuelas y universidades del Ecuador; sin embargo, la mayoría de los docentes tenemos componentes que podemos enseñar eficazmente a distancia. Estos son los contenidos que tradicionalmente enseñamos mediante conferencias, así como el no aprovechamiento de la interacción entre estudiantes y expertos dentro de un área específica del conocimiento propio de una materia. Descomponiendo así el muro que separa la institución educativa de la sociedad.

\section{Referencias Bibliográficas}

Barron, A. \&. Orwing. (2018). Multimedia technologies for training: An introduction. Englewood: CO: Libraries Unlimited.

Carver, C. \& Biehler. (2014). Incorporating multimedia and hypertext documents in an undergraduate curriculum. IEEE/ASEE Frontiers in education conference, 87-92.

Friel, D. \&. Knuth (2016). Learning with technology: Merging onto the information highway.

Oak Brook, IL: North Central Regional Educational Laboratory.

Kinzie, M. B. (2006). Frog dissection via the World-Wide Web: Implications for widespread delivery of instruction. Educational Technology Research and Development, 44(2), 59-69.

Seguin, A. (2017). Using the Internet in professional development. Dallas: Paper presented at the American Vocational Association Convention. (ERIC_ED 379 428). 